\title{
Disco de Poincaré: uma proposta para explorar geometria hiperbólica no GeoGebra
}

\author{
Ricardo Ribeiro Maria Gravina
}

\begin{abstract}
Resumo
Este artigo traz uma proposta de inserção de novo conteúdo na matemática escolar. Trata-se da exploração da geometria hiperbólica em ambiente de geometria dinâmica. Para isto foi feita a construção do micro-mundo Disco de Poincaré, usando-se o software GeoGebra. O menu construído permite a realização de atividades que tratam as ideias da geometria hiperbólica através de comparações com aquelas que são conhecidas e naturais na geometria euclidiana. $\mathrm{O}$ artigo apresenta o processo de construção do micro-mundo e também algumas atividades que foram concebidas para serem trabalhadas na escola; nele também são feitas considerações sobre a natureza da geometria e a sua evolução ao longo da história, de forma a contextualizar a proposta.
\end{abstract}

Palavras-chave: Geometria hiperbólica; geometria dinâmica; Disco de Poincaré.

Abstract This article deals with a didactic experience focusing on geometric transformations in the plane and use of the GeoGebra dynamic geometry environment. The experience, in the form of a workshop, was performed with elementary school teachers. Having as mathematical content the geometric transformations, the proposal integrated geometry and art through the construction of tessellation and Escher mosaics. The work was developed within the principles of Didactic Engineering and, for the accomplishment of the analyzes, we use the social-historical theory, whose main reference is the work of Vygotsky and also the theory of the records of representation by Duval, that deals with the process of learning of Mathematics from the point of view of semiotics. From the analysis, it was possible to verify that the participating teachers appropriated the resources and the system of representation that one has in GeoGebra, as well as the concepts of the geometry of the transformations.

Keywords: GeoGebra, Geometric transformations; Art; Dynamic geometry.

\section{Introdução}

Contemplar, no ensino escolar, o entendimento da geometria como um modelo teórico que pode estar além daquele estabelecido na geometria euclidiana, esse dependente de nossas experiências sobre o mundo sensível, pode propiciar, aos alunos, um olhar mais aguçado sobre a natureza da matemática.

Os conceitos geométricos deveriam ser parte importante do currículo de Matemática no Ensino Básico. Por meio deles o aluno desenvolve um tipo especial de pensamento - o pensamento geométrico - que lhe permite compreender, descrever e representar, de forma organizada o mundo em que vive. 
Algumas pesquisas ([2], [8], [10], [11]) que tratam do ensino de geometrias não-euclidianas na escola e na formação de professores, apontam para a importância de incorporar as geometrias não Euclidianas no currículo da Matemática escolar, salientando que os futuros professores devam ser preparados para seu ensino na escola. Alertam para a relevância da formação inicial do professor de Matemática como o ponto de partida para a efetivação de propostas que visam incluir as Geometrias não Euclidianas na Educação Básica. Enfatizam, também, a importância da utilização dos ambientes de geometria dinâmica e as interfaces de trabalho por eles disponibilizados, pois, os mesmos, propiciam a manipulação de objetos concretos-abstratos na tela do computador. Essa manipulação pode preparar o aluno na sua ascensão de patamar de conhecimento, de empírico para aquele inserido no modelo teórico que caracteriza uma geometria.

Neste artigo vamos apresentar uma proposta de ensino que tem como objetivo o entendimento das primeiras ideias de um modelo teórico que não se comporta como o modelo euclidiano. Nele não é mais válido o axioma das paralelas - aquele que garante que por um ponto exterior a uma reta passa uma e somente uma reta paralela. Agora, por um ponto exterior a uma reta têm-se infinitas retas paralelas e este é o axioma que caracteriza a geometria hiperbólica. A realização de atividades, no contexto desta geometria, pode ser uma fonte de enriquecimento cognitivo de nossos alunos - raciocinar com retas que não correspondem mais aquela ideia intuitiva usada na geometria euclidiana exige um novo patamar de abstração e de controle lógico de argumentos.

As atividades que projetamos para o estudo da geometria hiperbólica pressupõe um certo domínio da geometria euclidiana, entendendo-se aqui que são conteúdos que normalmente são trabalhados nos anos finais do ensino fundamental. Assim, consideramos que é uma proposta de ensino para alunos que estão cursando o ensino médio.

O assunto "geometria hiperbólica" é ausente nos livros didáticos. Isso faz sentido, se considerarmos que em apresentação no tradicional formato de texto escrito e figuras estáticas é um conteúdo que pode ser de difícil compreensão para alunos da escola básica. Nossa proposta se apoia, fortemente, no uso da geometria dinâmica, porque em um tal ambiente os alunos podem manipular, de forma concreta, os diferentes objetos do modelo hiperbólico - segmentos, retas, círculos, triângulos. Estamos admitindo que é através desta manipulação que, gradativamente, vão se constituindo as imagens mentais que concretizam uma nova possibilidade para a ideia de reta - um entendimento que é crucial na exploração desse novo espaço. Foi com esse princípio que construímos o micro-mundo Disco de Poincaré, com o software livre GeoGebra $(<w w w . g e o g e b r a . o r g>)$. E, fazendo uso deste micro-mundo, planejamos uma sequência de atividades de forma a provocar, nos alunos, a descoberta de semelhanças e diferenças entre a geometria euclidiana e a geometria hiperbólica.

No que segue, para contextualizar a proposta, iniciamos com uma breve retrospectiva sobre a natureza da geometria e a história da sua evolução. Depois apresentamos o micro-mundo Disco de Poincaré; e na última parte detalhamos algumas das atividades que foram projetadas para serem trabalhadas com alunos da escola básica. A proposta, na íntegra, está na dissertação de mestrado intitulada "Geometria NãoEuclidianas na Escola: uma proposta de ensino através da geometria dinâmica", apresentada pelo primeiro autor do artigo no Programa de Pós-Graduação em Ensino de Matemática do Instituto de Matemática da UFRGS.

\section{Sobre a natureza da geometria e a história da sua evolução}

Alguns historiadores $([1],[4])$ nos falam que a Geometria começou a se desenvolver através de necessidades relacionadas ao plantio, construções e movimento dos astros. Euclides foi o primeiro a apresentar a geometria organizada num encadeamento lógico-dedutivo, no qual cada proposição deveria ser deduzida de outra mais simples de maneira lógica e dedutiva. Em sua obra intitulada Elementos, ele reuniu praticamente todo o conhecimento de Matemática básica da sua época. Os Elementos, escrito por volta de 300 a.C, são compostos por 13 livros contendo 465 proposições. As dez afirmações inicias apresentadas por Euclides, 
na sua obra, se organizam em dois grupos: os axiomas e os postulados. Os antigos matemáticos faziam distinção entre axioma e postulado: axioma é uma noção comum aceitável como hipótese em qualquer ciência; postulado é hipótese própria da Geometria.

Durante quase dois mil anos, a geometria de Euclides foi considerada como a única geometria possível. No entanto, o quinto postulado - "se uma reta corta duas outras retas formando ângulos colaterais internos cuja soma é menor do que dois retos, então as duas retas, se continuadas infinitamente, encontram-se no lado onde estão os ângulos cuja soma é menor do que dois retos"- pelo fato de possuir uma redação mais complexa, extensa e menos intuitiva que os postulados anteriores se tornou motivo de forte questionamento nos séculos XVII e XVIII. Diferentes matemáticos, dentre eles John Wallis (1616 - 1703), Saccheri (1667 1733), Lambert (1728 - 1777), Legendre (1752 - 1833), fizeram suas tentativas de demonstração do quinto postulado. Para estes matemáticos o quinto postulado, conforme enunciado acima, era questionável por não ser intuitivamente óbvio que as duas retas em questão deveriam, de fato, se encontrar no infinito. E assim, o postulado começa a ser pensado como uma afirmação a ser demonstrada. Inúmeras foram as tentativas de demonstração sendo que muitas delas admitiam, nos argumentos, fatos equivalentes ao próprio postulado. Uma das consequências, que veio dessas tentativas de demonstração, foi a produção de vários postulados equivalentes ao quinto postulado, denominados de postulados substitutos.

O postulado substituto mais conhecido é o que foi apresentado pelo matemático escocês John Playfair (1748-1819) no seu trabalho Elementos de Geometria, publicado em 1795. Em linguagem moderna, o axioma de Playfair é apresentado na seguinte formulação: por um ponto fora de uma reta pode-se traçar uma única reta paralela à reta dada. Esse enunciado acabou batizando o quinto postulado com o nome de Postulado das Paralelas.

Uma das tentativas de demonstrar o quinto postulado foi feita por Girolamo Saccheri (1667 - 1733), em um livro chamado "Euclides ab omni naevo vindicatus" (Euclides, sem qualquer falha) publicado em 1733. Saccheri foi o primeiro matemático a considerar uma hipótese contraditória ao quinto postulado. Sendo ele detentor de um grande conhecimento de lógica, criou um quadrilátero, conhecido como "quadrilátero de Saccheri", o qual possuía dois ângulos retos e dois lados opostos de mesmo comprimento. Sua ideia era provar, a partir dos quatro primeiros axiomas, que os outros dois ângulos do quadrilátero também eram retos. Isso era equivalente a provar o quinto postulado. Todavia, Saccheri só conseguiu mostrar que os outros dois ângulos eram congruentes. Em sua busca ele obteve alguns resultados que depois vieram a fazer parte do corpo de propriedades da geometria não-euclidiana; mas no momento da descoberta, Saccheri os considerou tais resultados abomináveis por ferirem a intuição.

A história nos mostra que foram as tentativas de transformar o quinto postulado do sistema axiomático euclidiano em um teorema que desencadearam o desenvolvimento da geometria hiperbólica.

\subsection{A Geometria Hiperbólica}

Foi apenas na primeira metade do século XIX que se começou a suspeitar que o Postulado das Paralelas fosse realmente independente dos demais postulados. Matemáticos como Carl Friedrich Gauss (1777-1855), Johann Bolyai (1802-1860) e Nicolai Ivanovich Lobachewsky (1793-1856) trataram da questão ao considerar três situações distintas: por um ponto não contido em uma reta dada, passa mais de uma, apenas uma ou nenhuma reta paralela a reta dada. Por suspeitarem da independência do Postulado das Paralelas, ou seja, de que sua negação poderia gerar uma geometria consistente, sem contradições, desenvolveram, de forma axiomática, um estudo amplo e detalhado de uma geometria que assumia a existência de mais de uma reta paralela a uma dada reta, sendo assim lançada a semente do que viria a ser a Geometria de Lobatchevsky ou Geometria Hiperbólica.

A história nos diz que Gauss, Bolyai e Lobachewsky desenvolveram a Geometria Hiperbólica ao mesmo tempo ([3]). No entanto, Lobachewsky foi o primeiro a publicar seus trabalhos, cabendo a ele a honra da descoberta desta geometria que ele também chamou de Imaginária. 
De acordo com [1], o primeiro trabalho de Lobachewsky sobre Geometria não Euclidiana foi publicado, em 1829, no Kasan Bulletin. Nas palavras do historiador: ,

\begin{abstract}
Este artigo marca oficialmente o nascimento da Geometria Não-Euclidiana, pois foi Lobachewsky o primeiro matemático a dar o passo revolucionário de publicar uma Geometria especificamente construída sobre uma hipótese em conflito direto com o Postulado das Paralelas: Por um ponto $C$ fora de uma reta $A B$ pode-se traçar mais de uma reta do plano que não encontra $A B$. [1, p397]
\end{abstract}

No entanto, as dúvidas referentes à consistência da geometria hiperbólica, só foram dirimidas no final do século XIX, quando matemáticos como Eugenio Beltrami (1835-1900), Henri Poincaré (1854-1912) e Felix Klein (1849-1925) criaram no universo euclidiano modelos para essa nova geometria. Um modelo para um determinado sistema axiomático é uma interpretação dada aos conceitos primitivos de modo que os axiomas sejam todos propriedades verdadeiras. Vamos aqui falar do modelo do disco de Poincaré, pois é nele que se apoia a nossa proposta de ensino.

No modelo do Disco de Poincaré, o plano hiperbólico é definido a partir da região limitada por uma circunferência. Denominamos essa região de Disco. Os pontos internos a esta circunferência são denominados pontos do plano hiperbólico; os pontos que pertencem à circunferência são denominados pontos ideais e a circunferência é dita horizonte hiperbólico. Os arcos de circunferência contidos no Disco e ortogonais ao horizonte hiperbólico são as retas hiperbólicas (Figura 1). Neste modelo, por um ponto P exterior a uma reta $\mathrm{r}$ passam infinitas retas paralela à $\mathrm{r}$ (Figura 2). E também temos que a soma dos ângulos internos de um triângulo é sempre menor do que dois ângulos retos; quando o triângulo é pequeno, seus ângulos somam aproximadamente $180^{\circ}$.

As figuras abaixo ilustram estes primeiros conceitos e propriedades da geometria hiperbólica.

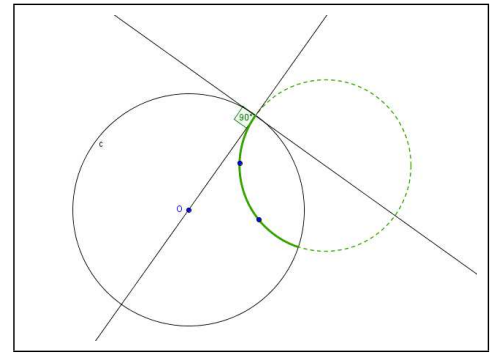

Figura 1: Reta hiperbólica como arco de circunferência ortogonal ao horizonte.

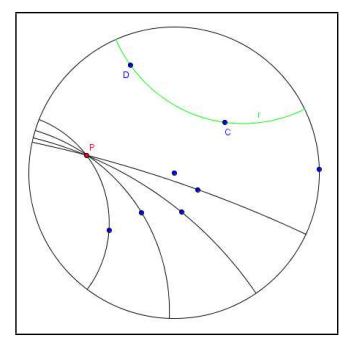

Figura 2: Infinitas retas hiperbólicas paralelas a $\mathrm{r}$ passando por $\mathrm{P}$.

Para calcular a distância hiperbólica entre os pontos A e B, traçamos a reta hiperbólica que passa por esses pontos e consideramos os pontos $\mathrm{C}$ e D que estão na circunferência euclidiana que define o Disco de Poincaré (Figura 3). A partir da Figura 3 pode-se estabelecer a seguinte relação: $d(A, B)=\left|\ln \left(\frac{A C / A D}{B C / B D}\right)\right|$. 


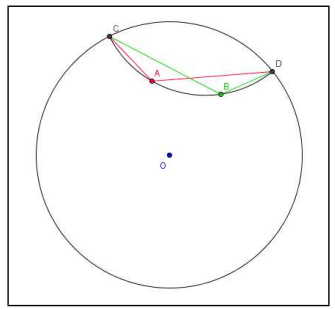

Figura 3: Razão cruzada.

As medidas AC, BC, AD e BD correspondem às medidas de segmentos euclidianos e a partir dessa definição, podemos fazer as seguintes observações:

- quando o ponto A tende ao ponto $\mathrm{C}$ (se aproxima do horizonte), temos que a distância euclidiana AC tende a zero. Com isso a razão tende a zero e o logaritmo da razão tende ao infinito negativo. Como a expressão está em módulo, à distância hiperbólica entre os pontos A e B tende ao infinito.

- quando o ponto B tende ao ponto D (se aproxima do horizonte), temos que a distância euclidiana BD tende a zero. Com isso a razão tende ao infinito e o logaritmo da razão também tende ao infinito, Ou seja, a distância hiperbólica entre os pontos A e B tende ao infinito.

- quando o ponto $\mathrm{B}$ tende ao ponto $\mathrm{A}$, temos que $\mathrm{BD}$ tende a $\mathrm{AD}$. Com isso a razão tende a 1 e o logaritmo da razão tende a zero. Ou seja, a distância hiperbólica entre os pontos A e B tende a zero.

É importante entender, de forma intuitiva, esta noção de distância no Disco. O Disco é um espaço infinito, no seguinte sentido: um ser habitando este mundo bidimensional pode caminhar na direção do horizonte, com passos de mesmo tamanho, sem nunca chegar ao fim de sua caminhada. Um observador externo vê os seus passos irem se tornando cada vez menores, mas isto é uma distorção para quem está olhando o caminho hiperbólico com "olhos euclidianos". Na Figura 4 temos os primeiros passos do tal ser, depois um zoom para ver que a partir do ponto que ele está outros tantos passo iguais podem ser dados. Poderíamos repetir o procedimento de zoom indefinidamente e sempre vamos ver que outros tantos passos iguais podem ser dados, sem que haja uma aproximação do horizonte. Vale ainda observar que os segmentos que compõem o caminho ilustrado na Figura 4 possuem o mesmo comprimento hiperbólico, embora aos "olhos euclidianos"pareçam ter comprimentos diferentes.

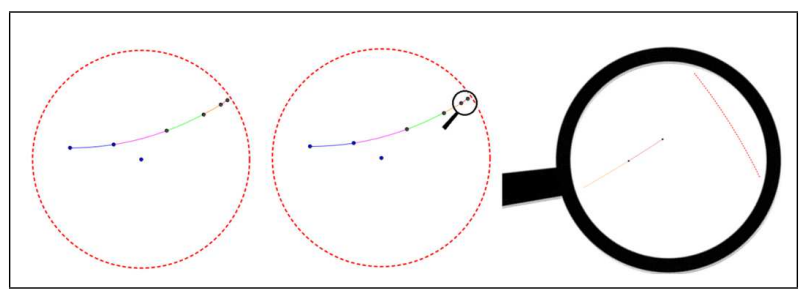

Figura 4: Razão cruzada.

Pensar em um mundo diferente do euclidiano exige um desprendimento da experiência sensível imediata e, consequentemente, também exige controle racional das propriedades geométricas que valem nesse novo mundo. Pode-se dizer que é nas experiências no mundo sensível imediato que se constroem as ideias 
intuitivas que dão suporte ao entendimento de propriedades geométricas euclidianas. No caso da geometria hiperbólica é através de um micro-mundo dinâmico que vamos propor a realização de experiências que vão tornar familiar as ideias que constituem esta outra geometria.

\section{A construção do micro-mundo "Disco de Poincaré"}

Nos softwares de geometria dinâmica, o processo de construção das figuras é feito mediante o uso de menus em linguagem natural da geometria, como por exemplo, ponto, reta passando por dois pontos, retas paralelas, retas perpendiculares, círculos. Mas, tais softwares oferecem muito mais do que a possibilidade de efetuar construções geométricas de modo rápido e preciso. Eles oferecem a possibilidade de movimentar as figuras geométricas, mudando tamanho e posição, mas guardando as propriedades geométricas que as caracterizam. Este recurso, que é referido como "estabilidade sob ação do movimento", propicia explorações que provocam a concretização de ideias matemáticas ([5], [6]). É apostando nesta característica do GeoGebra que construímos o micro-mundo Disco de Poincaré.

Para construir este micro-mundo utilizamos, de forma intensa, o recurso Criar uma Nova Ferramenta do GeoGebra. As diferentes ferramentas do menu hiperbólico são resultantes de construções feitas na geometria euclidiana e que foram automatizadas através deste recurso. A Figura 5 mostra a interface do micro-mundo. Os ícones correspondentes as ferramentas são imagens em formato jpg, que é suportado pelo GeoGebra.

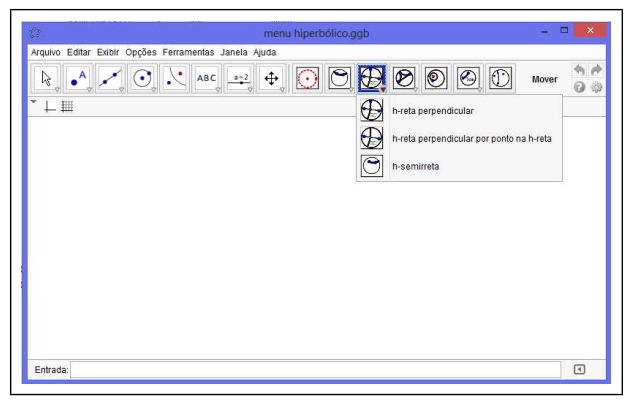

Figura 5: Interface GeoGebra com menu hiperbólico.

Na Tabela 1 apresentamos as principais ferramentas disponibilizadas no micro-mundo. 


\begin{tabular}{|c|c|c|}
\hline Ícone & Recurso & Função \\
\hline & Disco & $\begin{array}{l}\text { Constrói o Disco com borda pontilhada indicando-se o } \\
\text { centro e um dos pontos da borda. }\end{array}$ \\
\hline 0 & h-reta & $\begin{array}{l}\text { Constrói a reta hiperbólica indicando-se o Disco e dois } \\
\text { pontos. }\end{array}$ \\
\hline$\Theta$ & $\begin{array}{l}\text { h-reta (pontos da } \\
\text { borda) }\end{array}$ & $\begin{array}{l}\text { Constrói a reta hiperbólica com os pontos ideias (inter- } \\
\text { secção da reta com o Disco) indicando-se o Disco e dois } \\
\text { pontos. }\end{array}$ \\
\hline (อ) & h-segmento & $\begin{array}{l}\text { Constrói o segmento hiperbólico indicando-se o Disco e } \\
\text { dois pontos. }\end{array}$ \\
\hline (9) & h-círculo & $\begin{array}{l}\text { Constrói o círculo hiperbólico indicando-se o Disco e dois } \\
\text { pontos. }\end{array}$ \\
\hline & h-triângulo & $\begin{array}{l}\text { Constrói o triângulo hiperbólico indicando-se o Disco e } \\
\text { três pontos. }\end{array}$ \\
\hline (8) & h-ângulo & $\begin{array}{l}\text { Determina a medida do ângulo hiperbólico indicando-se } \\
\text { o Disco e três pontos. }\end{array}$ \\
\hline (D) & h-reflexão ponto & $\begin{array}{l}\text { Constrói a reflexão de um ponto hiperbólico em relação } \\
\text { a uma reta hiperbólica indicando-se Disco, reta hiper- } \\
\text { bólica e ponto. }\end{array}$ \\
\hline
\end{tabular}

Tabela 1: Ferramentas hiperbólicas.

No que segue, para ilustrar o uso do recurso Criar uma Nova Ferramenta do GeoGebra, vamos apresentar o procedimento de construção de uma das ferramentas do menu hiperbólico. Escolhemos fazer a construção da $h$-reta hiperbólica, pois no procedimento tem-se também interessante propriedade da geometria euclidiana.

De início construímos o Disco de centro A passando por $\mathrm{B}$, usando Círculo dado centro e um de seus pontos, e dando-lhe o destaque de borda pontilhada em vermelho para indicar o horizonte hiperbólico. O Disco é o conjunto de pontos que estão no interior do círculo pontilhado.

Tendo-se o Disco e dois de seus pontos C e D, o problema que se apresenta é como construir uma circunferência ortogonal ao horizonte do Disco, passando por C e D. Relembramos aqui que uma reta hiperbólica, que vamos referir como h-reta, é um arco de tal círculo ortogonal ao horizonte do Disco.

Adiantamos que é no menu das transformações geométricas, disponível no GeoGebra, que vamos encontrar a solução do problema - trata-se da transformação Reflexão em Relação a um Círculo (Inversão). Sobre esta transformação: dado um círculo de centro em $\mathrm{O}$ e raio $\mathrm{r}$, dizemos que o inverso do ponto $\mathrm{P}$ em relação ao círculo é o ponto P' sobre a semirreta OP que satisfaz a relação OP'.OP = r.r.

No GeoGebra tem-se, no menu das transformações, essa transformação de Inversão. A Figura 6 apresenta a construção geométrica que produz a Inversão. Apartir da semelhança dos triângulos retângulos OPD e ODP' é fácil obter a igualdade de razões que implica que OP'.OP = r.r . 


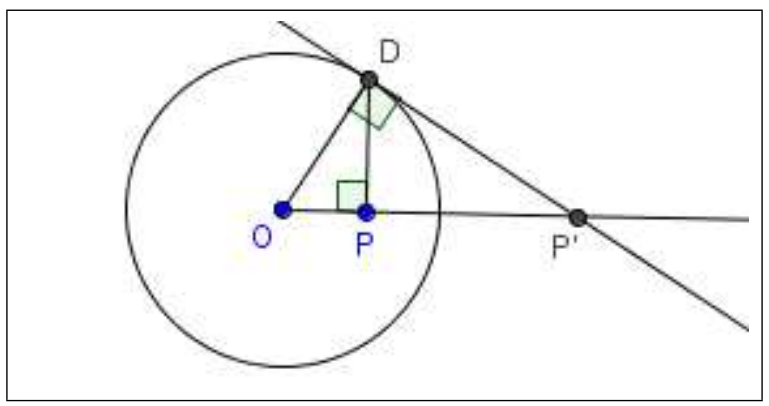

Figura 6: Ponto $\mathrm{P}^{\prime}$ inverso do ponto $\mathrm{P}$.

É o teorema que diz: "Se $P$ e $P$ ' são pontos inversos um do outro em relação ao horizonte hiperbólico, então qualquer círculo passando por $P$ e $P^{\prime}$ é ortogonal ao horizonte hiperbólico" que resolve o problema de construção da h-reta. A demonstração deste teorema pode ser vista em [9].

Assim, tendo-se o Disco e seus dois pontos C e D, o procedimento de construção da h-reta é:

- aplicamos a Reflexão em Relação a um Círculo (Inversão) no ponto C, aqui tomando o círculo pontilhado que é o horizonte hiperbólico, e obtemos o ponto refletido C'.

- aplicamos o menu Círculo definido por Três Pontos nos pontos C, C' e D.

- para a construção do arco que corresponde a h-reta, marcamos os pontos E e F de intersecção dos dois círculos.

- aplicamos Mediatriz nos pontos E e F. Após marcamos o ponto G de intersecção da reta mediatriz com o círculo ortogonal já construído. É importante a construção desta mediatriz para garantir que o arco que definirá a h-reta fique no interior do círculo pontilhado.

- aplicamos Arco Circular definido por Três Pontos nos pontos E, G e F.

- aplicamos Exibir/Esconder Objeto nos pontos C', E, F e G, na reta mediatriz e no círculo ortogonal.

Os passos de construção apresentados acima estão ilustrados na Figura 7

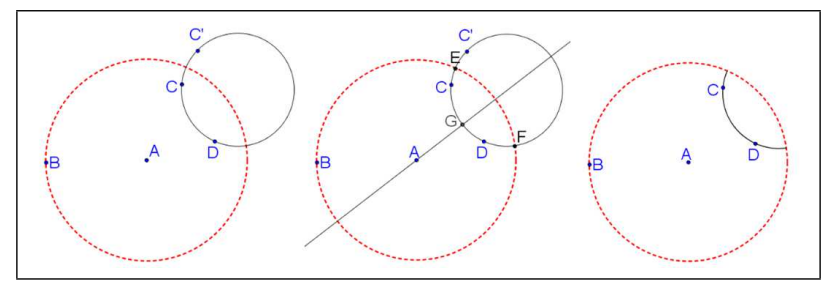

Figura 7: Construção da h-reta.

Feita a construção da $h$-reta, iniciamos o processo de Criar uma Nova Ferramenta e assim automatizamos o procedimento de construção apresentado acima. Conforme ilustra a Figura 8: a) escolhemos como objeto final o arco de círculo perpendicular ao horizonte hiperbólico; b) como objetos iniciais escolhemos o Disco e os pontos C e D. 


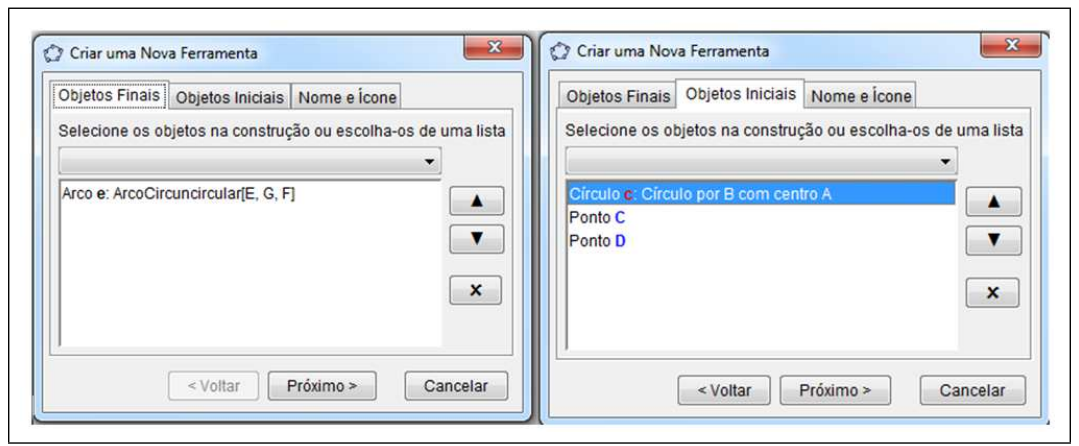

Figura 8: Janela Objetos Finais e Objetos Iniciais da ferramenta h-reta.

Todos as ferramentas disponíveis no micro-mundo Disco de Poincaré foram construídas desta mesma forma. Segundo [6] tem-se "na tecnologia digital a ampliação das possibilidades para experimentos de pensamentos... Essa tecnologia disponibiliza, cada vez mais, ferramentas que suportam a exteriorização, a diversificação e a ampliação de pensamentos". Na construção do micro-mundo levamos em consideração tal potencial. Na próxima seção vamos apresentar as possibilidades que este menu oferece para a exploração de ideias no mundo hiperbólico.

\section{O Disco de Poincaré e possibilidades para o ensino}

Já no momento de construção do micro-mundo Disco de Poincaré estávamos pensando em atividades que poderiam ser desenvolvidas na escola. A escolha das atividades teve como referência as noções básicas e bem conhecidas da geometria euclidiana e tomou como pressuposto que os ambientes de geometria dinâmica favorecem os experimentos, de forma tal que as figuras na tela do computador tornam-se objetos concreto-abstratos: concretos porque podem ser manipulados diretamente, e abstratos porque tratam de veicular ideias tais como reta hiperbólica, triângulo hiperbólico, paralelismo e perpendicularidade no mundo hiperbólico.

No processo de aprendizagem da geometria um dos aspectos centrais é a passagem do empírico para o dedutivo. De acordo com a teoria de Van Hielle (1950), podemos dizer que são os níveis elementares da visualização e da análise que preparam para os níveis mais avançados da dedução informal e dedução formal. Na proposta de ensino que vamos apresentar, em se tratando de geometria não-euclidiana na escola, nosso objetivo é o desenvolvimento de habilidades que estão nos dois primeiros níveis propostos por Van Hielle: a visualização e a análise.

É com as explorações empíricas no Disco de Poincaré que se pretende o entendimento de que a ideia de reta não precisa estar associada com aquela construída na experiência no mundo físico imediato - aqui estamos nos referindo a ideia com que trabalhamos na geometria euclidiana. E também se pretende o entendimento de que a soma dos ângulos de um triângulo depende do "mundo" em que este triângulo se encontra, ou seja, depende da geometria. No caso da geometria hiperbólica, as explorações vão mostrar que a soma dos ângulos pode variar entre $0^{\circ}$ e $180^{\circ}$.

Abaixo discutimos quatro das atividades que estão na proposta de ensino, na sua íntegra detalhada em [9]; as três primeiras tratam de conceitos fundamentais e a última, com enfoque artístico, trata de pavimentação hiperbólica. Na íntegra da proposta, têm-se atividades que, gradativamente, vão introduzindo as ideias da geometria hiperbólica e para cada uma delas tem-se comentário que destaca o objetivo quanto à aprendizagem dos alunos, acompanhado de figuras ilustrativas. 
Aprimeira atividade tem o seguinte enunciado:

- Quantas h-retas passam por dois pontos A e B do Disco? Movimente o ponto A e observe o comportamento da h-reta.

- Dado uma h-reta e um ponto P que não pertence a ela, quantas h-retas passam por $P$ e não interceptam a h-reta dada?

\section{- Como podem ser as h-retas paralelas?}

O objetivo da atividade é que os alunos comecem a se habituar com uma nova ideia de reta. Eles podem observar que, assim como na geometria euclidiana, na geometria hiperbólica por um ponto passam infinitas h-retas e por dois pontos passa somente uma h-reta (Figuras 9 e 10). Movimentando pontos que estão na h-reta, eles podem observar variações na sua forma, sendo uma situação extrema o caso em que a h-reta é um diâmetro do Disco (Figura 11).

No terceiro item, tem-se a situação em que por um ponto exterior a uma h-reta passam infinitas h-retas paralelas (Figura 10). Ou seja, o quinto postulado da geometria euclidiana - o axioma das paralelas - não é válido na geometria hiperbólica.

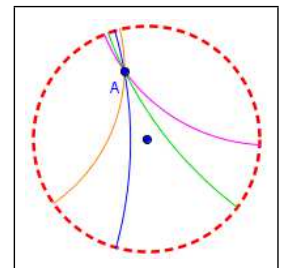

Figura 9: Retas hiperbólicas passando pelo ponto A.

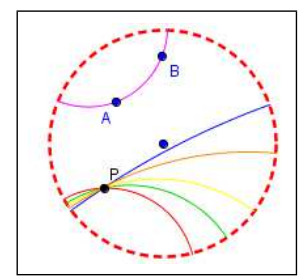

Figura 10: Retas hiperbólicas passando por P.

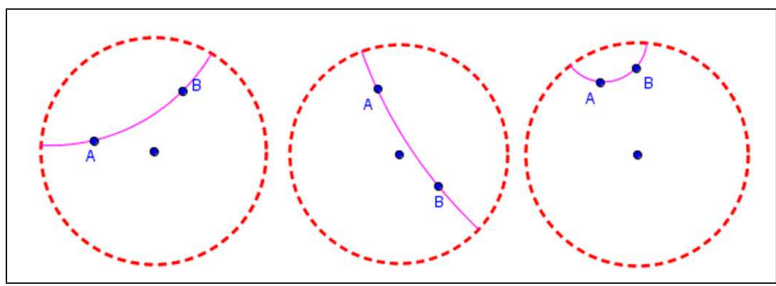

Figura 11: Reta hiperbólica passando por A e por B.

A segunda atividade propõe a exploração do conceito de distância entre pontos e tem o seguinte enunciado: Usando o menu h-circulo (círculo hiperbólico), construa o caminho de uma criatura, que se move no Disco com passos de mesmo tamanho. Quando, ao nosso olhar, o caminho se parece com um caminho euclidiano?

Nas Figuras 12 e 13 temos dois destes caminhos, ambos como mesmo número de passos. Aos nossos olhos euclidianos, o primeiro caminho surpreende, pois sendo feito com passos de mesmo tamanho, não é isto que vemos na figura. Já o segundo caminho registra os passos de mesmo tamanho, pois é um caminho que está próximo do centro do Disco, e nesta região a geometria hiperbólica se aproxima da geometria euclidiana. 


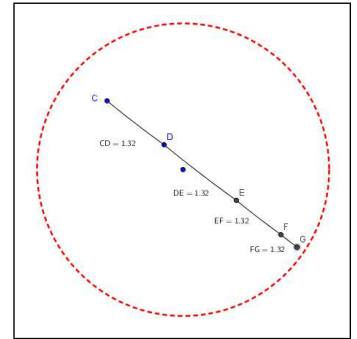

Figura 12: Segmentos hiperbólicos em sequência com os pontos distantes.

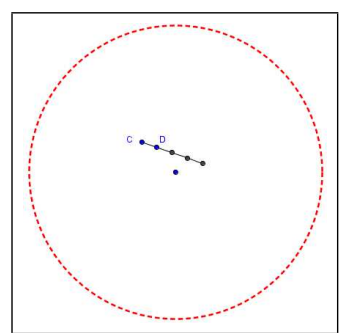

Figura 13: Segmentos hiperbólicos em sequência com os pontos próximos.

A terceira atividade propõe a exploração da medida de $h$-ângulo e da soma dos $h$-ângulos de um $h$ - triângulo, e tem o seguinte enunciado:

- O conhecido procedimento de construção do triângulo equilátero funciona no Disco? Quando o h-triângulo equilátero se parece com um triângulo euclidiano?

- Como se comporta a medida dos h-ângulos de um h-triângulo equilátero?

- Como construir um h-triângulo isósceles? Os h-ângulos da base do h-triângulo são congruentes entre si? Movimente os h-vértices e observe as formas possíveis para um h-triângulo isósceles.

Na primeira atividade é retomado o procedimento usual de construção de um triângulo equilátero da geometria euclidiana. Conforme ilustra a Figura 14, na geometria hiperbólica, a aparência do h-triângulo surpreende. Ao movimentar os seus vértices, o aluno pode observar que ele se parece com o triângulo equilátero euclidiano quando os vértices estiverem próximos do centro do Disco. Na segunda atividade, é possível obter um h-triângulo equilátero com soma das medidas dos h-ângulos muito próximo de zero, um outro fato surpreendente. O procedimento de construção do h-triângulo isósceles, também mostra que a aparência do $h$ - triângulo pode ser estranha. Essa construção pode ser feita a partir do h-círculo ou a partir da h-mediatriz.

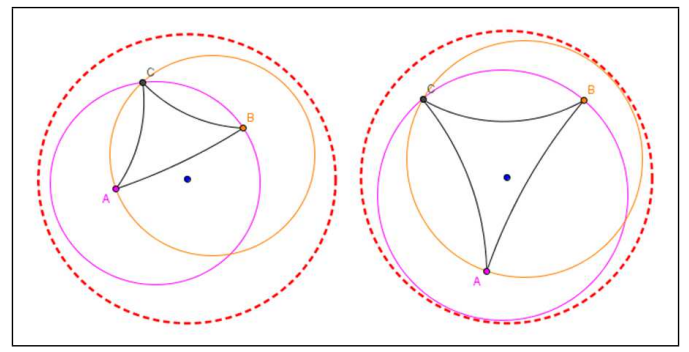

Figura 14: Construção de h-triângulo equilátero a partir da intersecção dos h-círculos. 


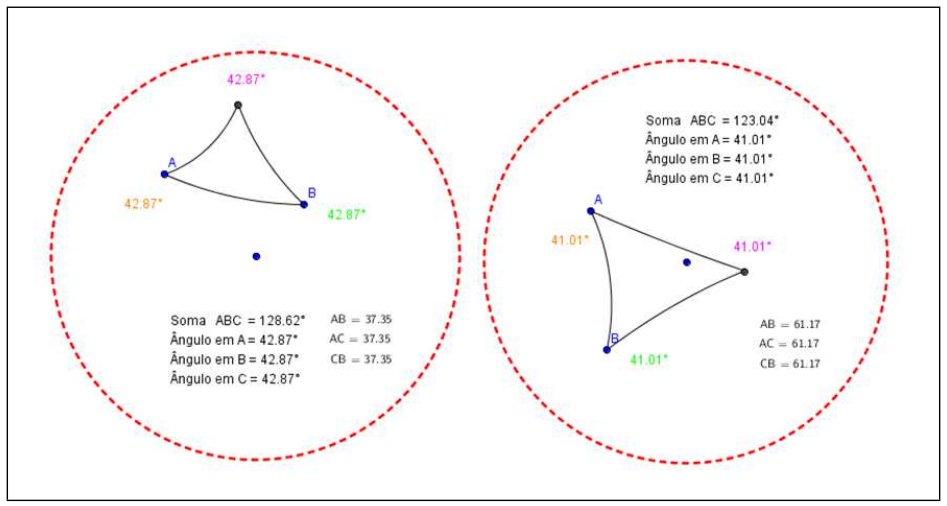

Figura 15: Medida da soma dos ângulos de h-triângulo equilátero.

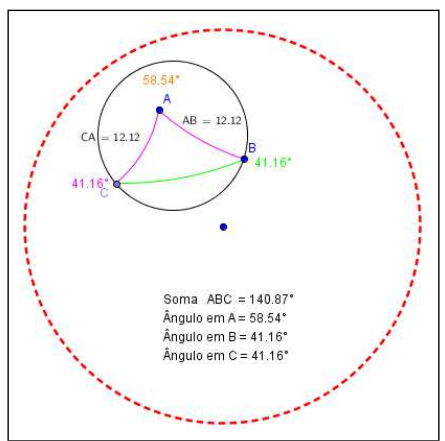

Figura 16: Construção de h-triângulo isósceles e soma dos seus h-ângulos.

A última atividade que vamos apresentar tem um caráter artístico. Usando a transformação de $h$-reflexão segundo uma h-reta se pode produzir pavimentações no Disco.

A transformação de $h$-reflexão funciona da mesma forma que a transformação de reflexão segundo uma reta na geometria euclidiana. A Figura 17 ilustra o procedimento de construção que resulta na h-reflexão de um ponto. 


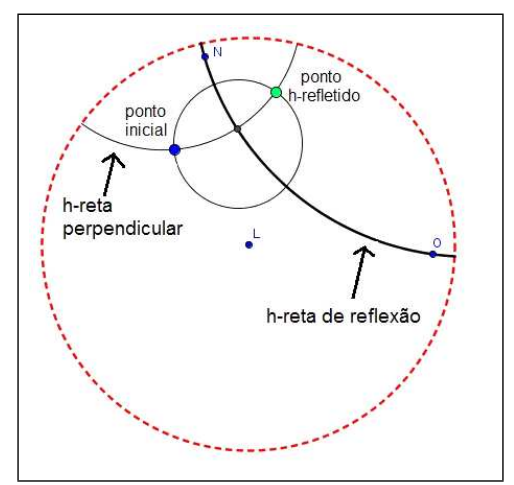

Figura 17: Reflexão de um ponto por uma h-reta.

As obras de Escher podem se tornar fonte de inspiração para a construção de pavimentações. São as propriedades do mundo da geometria hiperbólica que explicam os efeitos que podemos ver em duas de suas obras - Círculo Limite I e Círculo Limite III - ilustradas nas Figuras 18 e 19. Na segunda obra vê-se claramente, centralizada no Disco, uma composição feita com um h-quadrilátero regular e quatro h-triângulos equiláteros. Observe que esta mesma composição está sempre se repetindo e conforme a composição vai se aproximando do horizonte ela vai parecendo cada vez menor, ao nosso olhar euclidiano. No entanto, os h-quadriláteros são todos congruentes entre si, bem como os h-triângulos equiláteros, pois a composição é obtida através da transformação de h-reflexão. Este é o mundo da geometria hiperbólica.

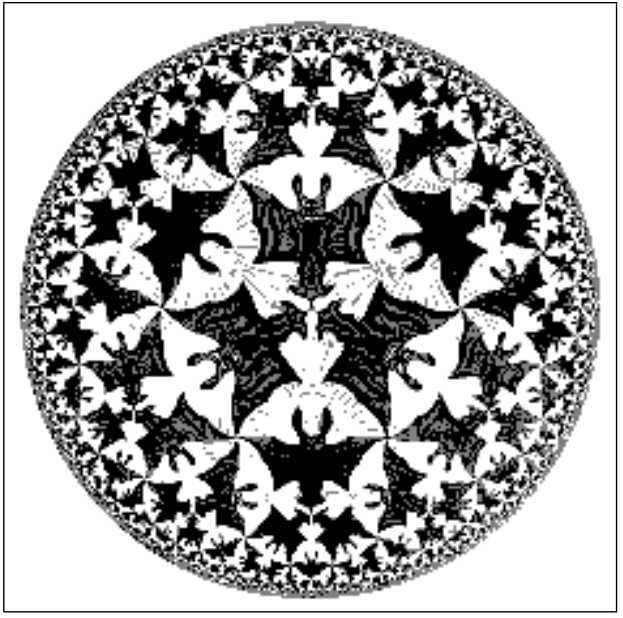

Figura 18: Círculo Limite I.

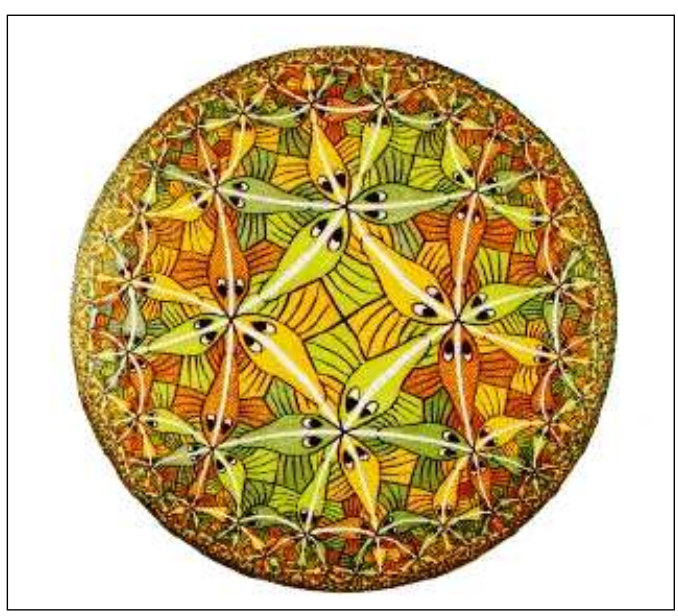

Figura 19: Círculo Limite III.

Abaixo temos uma figura produzida, no micro-mundo Disco de Poincaré, a partir de um h-triângulo equilátero. Ela inicia com o h-triângulo centralizado e segue com a sua $h$-reflexão segundo as três $h$-retas suportes dos seus lados. Estas reflexões geram novos $h$-triângulos equiláteros aos quais se aplicam novos procedimentos de h-reflexão, e assim sucessivamente, de modo a obter a Figura 20. Os efeitos de cores, obtidos na Figura 21, foram trabalhados em um editor de imagem. 


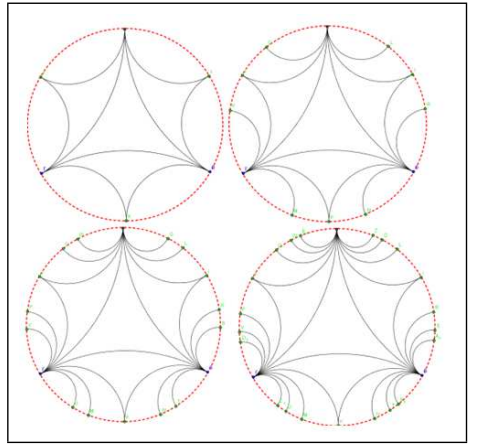

Figura 20: Passos de construção da pavimentação.

\section{Considerações finais}

Este artigo é um recorte do trabalho de [9]. Nele, um pressuposto fundamental é quanto ao uso da geometria dinâmica na aprendizagem da geometria hiperbólica: a manipulação das figuras dinâmicas, na tela do computador, é de fundamental importância no processo de aprendizagem de ideias que se confrontam com aquelas já construídas na geometria euclidiana.

O trabalho foi realizado no âmbito do programa de mestrado profissionalizante, e assim estamos disponibilizando um produto didático que pode ser utilizado por outros professores de matemática. Este produto é o micro-mundo Disco de Poincaré, disponível para download em <www.mat.ufrgs.br/ ppgem/produto didatico/rribeiro $>$. Além da construção do micro-mundo também elaboramos uma sequência de atividades, com o objetivo de provocar nos alunos muitos experimentos de pensamento, via manipulação direta na tela do computador, e sempre em paralelo com conceitos e ideias já conhecidos da geometria euclidiana. O conhecimento e a compreensão da geometria hiperbólica pode ajudar os alunos na construção do pensamento geométrico, e de uma forma desafiadora, pois as impressões visuais precisam ser colocadas sob controle racional.

Finalizando, diríamos que nossa proposta de introdução à geometria hiperbólica através da geometria dinâmica pode ser uma contribuição na direção de um ensino diferenciado da geometria escolar. Fica aqui o convite ao professor-leitor para que teste e aprimore o material didático que estamos colocando a disposição.

\section{Referências}

[1] Boyer, C. B. História da Matemática. São Paulo: Editora Edgard Blücher, 1996.

[2] Cabariti, E. Geometria Hiperbólica: uma proposta didática em ambiente informatizado. Dissertação de Mestrado em Educação Matemática, Pontifícia Universidade Católica de São Paulo, 2004.

[3] Eves, H. Introdução à História da Matemática. Tradução de Hygino H. Domingues. São Paulo: Editora Unicamp, p. 844, 2002

[4] Eves, H. Tópicos de História da Geometria. São Paulo: Atual, 1992.

[5] Gravina, M. A. Os Ambientes de Geometria Dinâmica e o Pensamento Hipotético-Dedutivo. Tese de Doutorado, Universidade Federal do Rio Grande do Sul, 2001.

[6] Gravina, M.A. et al. Matemática, mídias digitais e didática : tripé para formação de professores de matemática. Porto Alegre: Evangraf, 2012. Disponível em: <http://www6.ufrgs.br/espmat/livros/ livro2-matematica_midiasdigitais_didatica.pdf>. Acesso em: 19 de julho de 2013. 
[7] Pataki, I. Geometria Esférica para a Formação de Professores: Uma proposta interdisciplinar. Dissertação de Mestrado em Educação Matemática, Pontifícia Universidade Católica de São Paulo, 2003.

[8] Ribeiro, R. D. G. O ensino das geometrias não-euclidianas: um olhar sob a perpectiva da divulgação cientifica. Dissertação de Mestrado Profissionalizante em Educação Matemática, Faculdade de Educação da Universidade de São Paulo, 2012.

[9] Ribeiro, R. S. Geometrias Não-Euclidianas na Escola: Uma proposta de Ensino Através da Geometria Dinâmica. Dissertação de Mestrado, Universidade Federal do Rio Grande do Sul, 2013. Disponível em: <http://www.lume.ufrgs.br/>.

[10] Reis, J. D. S. Geometria Esférica por meio de Materiais Manipuláveis. Dissertação de Mestrado em Educação, UNESP, Rio Claro, SP, 2006.

[11] Zulatto, R. B. A. Professores de matemática que utilizam softwares de geometria dinâmica: suas características e perspectivas. Dissertação de Mestrado. UNESP, Rio Claro, SP, 2006.

Ricardo Ribeiro

Instituto Federal de Educação, Ciências e Tecnologia do RGS <ricardo.ribeiro@canoas.ifrs.edu.br>

Maria Gravina Instituto de Matemática da UFRGS

<gravina@mat.ufrgs.br>

Recebido: 2013

Publicado: 2013 